\title{
A TRANSIENT POTASSIUM CONDUCTANCE REGULATES THE EXCITABILITY OF CULTURED HIPPOCAMPAL AND SPINAL NEURONS ${ }^{1}$
}

\author{
MENAHEM SEGAL, ${ }^{2}$ MICHAEL A. ROGAWSKI, ${ }^{3}$ AND JEFFERY L. BARKER \\ Laboratory of Neurophysiology, National Institute of Neurological and Communicative Disorders and Stroke, National Institutes \\ of Health, Bethesda, Maryland 20205
}

Received July 27, 1983; Revised September 26, 1983; Accepted October 3, 1983

\begin{abstract}
Cultured mouse spinal cord and rat hippocampal neurons were voltage clamped with a twoelectrode system in the presence of tetrodotoxin $(1$ to $3 \mu \mathrm{M})$ and tetraethylammonium (25 mM). Depolarizing voltage commands from holding potentials negative to $-50 \mathrm{mV}$ evoked a transient outward current response that peaked within $10 \mathrm{msec}$ and decayed exponentially with a time constant of 10 to $40 \mathrm{msec}$. The current was inactivated by holding at potentials more positive than $-50 \mathrm{mV}$ but could be reactivated by brief hyperpolarizing prepulses $(20$-msec steps to $-90 \mathrm{mV}$ reactivated $50 \%$ ). The inversion potential of the tail current evoked in a double-step protocol was -70 to $-75 \mathrm{mV}$, and this shifted in a depolarizing direction with elevated $\left[\mathrm{K}^{+}\right]_{0}$. The transient outward current was blocked by external application of 4-aminopyridine (4-AP) with an $\mathrm{IC}_{50}$ of 2 $\mathrm{mM}$. In experiments under current clamp, 4-AP suppressed an initial transient rectification of the electrotonic potential recorded in response to depolarizing current and reduced the threshold for single and repetitive action potentials. Thus, a transient $\mathrm{K}^{+}$conductance similar to $I_{A}$ of molluscan neurons may serve to regulate the excitability of some central mammalian neurons. Depression of this conductance by 4-AP could contribute to the drug's convulsant effects in vivo.
\end{abstract}

Neuronal membranes possess several types of voltagedependent potassium conductance (Adams et al., 1980). One of these conductances, termed $I_{A}$ by Connor and Stevens (1971a), is transiently activated by depolarizing voltage steps from potentials more negative than the resting potential. It is thought to regulate the frequency of repetitive action potential firing (Conner and Stevens, 1971b), to alter synaptic output (Shimahara, 1981), and to be modified by associative learning in photoreceptors (Alkon et al., 1982). Recently, $I_{A}$ has been observed in two vertebrate neurons, sympathetic ganglion cells (Adams et al., 1982b; Galvan, 1982) and guinea pig hippocampal neurons (Gustafsson et al., 1982). Using a twoelectrode voltage clamp system, we have identified a transient voltage-dependent $\mathrm{K}^{+}$conductance in neurons

\footnotetext{
${ }^{1}$ We thank Joann Mazzetta for assistance with the cultures. M. A. R. is a Medical Staff Fellow in the Pharmacological Sciences Program, National Institute of General Medical Sciences.

${ }^{2}$ Present address: Isotope Department, The Weizmann Institute, Rehovot, Israel.

${ }^{3}$ To whom correspondence should be addressed, at Laboratory of Neurophysiology, National Institute of Neurological and Communicative Disorders and Stroke, Building 36, Room 2C-02, Bethesda, MD 20205.
}

cultured from embryonic mammalian spinal cord and hippocampus. In the present series of experiments we defined the characteristics of this conductance and explored its role in regulating the excitability of these cells.

Preliminary reports of these data have appeared previously (Barker and Rogawski, 1982; Segal and Barker, 1982).

\section{Materials and Methods}

Spinal cords from 13-day-old C57BL/6 mouse embryos or hippocampi from 18- to 19-day Sprague-Dawley rat embryos were dissected and grown in culture as previously described (Ransom et al., 1977; Barker et al., 1982; Segal, 1983). At the time of experiment the cultures were placed on the modified stage of an inverted phase microscope and bathed in Hank's balanced salt solution buffered to $\mathrm{pH} 7.4$ with $5 \mathrm{~mm}$ HEPES.

To separate $I_{A}$ from the voltage-dependent $\mathrm{Na}^{+}$and delayed $\mathrm{K}^{+}$currents, recordings were made in solutions containing tetrodotoxin (TTX; 1 to $3 \mu \mathrm{M}$; Sigma Chemical Co., St. Louis, MO) and tetraethylammonium chloride (TEA; $25 \mathrm{mM}$; various suppliers). When TEA was added to the medium, the concentration of $\mathrm{NaCl}$ was reduced to maintain the osmolality at $310 \mathrm{mOsm} / \mathrm{liter}$. 
On some occasions $\mathrm{CdCl}_{2}(0.2 \mathrm{mM})$ or $\mathrm{CoCl}_{2}(5 \mathrm{~mm})$ was added to the bathing solution to block $\mathrm{Ca}^{2+}$ current.

Solutions of drugs and salts were applied by pressure ejection $(<1 \mathrm{psi})$ from blunt micropipettes with tips broken back to $<2$ to $3 \mu \mathrm{m}$ diameter. During periods of drug ejection, the pipettes were situated close to the cell membrane, but, to avoid effects of drug diffusion, the pipettes were otherwise kept away from the cell. Concentrated drug solutions were prepared fresh daily and diluted to the appropriate final concentration in the bathing medium. The $\mathrm{pH}$ of the drug solution was monitored continuously with phenol red $(5 \mathrm{mg} / \mathrm{liter})$; when drugs changed the $\mathrm{pH}$, the solution was readjusted to 7.4 .

Recordings were made at room temperature (21 to $24^{\circ} \mathrm{C}$ ) from 4 - to 6 -week-old, pyramidal-shaped hippocampal cells ( 15 to $20 \mu \mathrm{m}$ diameter cell body) and from 2- to 3-month-old spinal cord cells (20 to $40 \mu$ m diameter cell body). Neurons were impaled with two microelectrodes filled with $3 \mathrm{M} \mathrm{KCl}$, and membrane potential was controlled using a two-electrode voltage clamp system (Smith et al., 1980). Electrodes were pulled on a BrownFlaming micropipette puller (model P-77, Sutter Instrument Co., San Francisco, CA) adjusted to produce tip resistances of 50 to 80 megohms. Both electrodes were shielded with aluminum foil wrapped along their shafts. The fluid level in the bath was kept low to reduce capacitative coupling between the electrodes. The settling time of the voltage signal following a $40-\mathrm{mV}$ command usually did not exceed $1 \mathrm{msec}$.

Current and voltage signals were displayed on a Brush chart recorder, and stored in a Nicolet digital oscilloscope for plotting on an $\mathrm{x}-\mathrm{y}$ recorder. Activation and inactivation curves were constructed by methods described by Thompson (1977).

\section{Results}

Stable recordings were obtained from 63 hippocampal neurons and 35 spinal cord cells. With double-electrode penetrations the average input resistances $( \pm$ SEM) of spinal and hippocampal cells measured at $-70 \mathrm{mV}$ were $23.1 \pm 1.9$ megohms and $29.6 \pm 1.7$ megohms, respectively. The membrane potential of the cells was held in the range -60 to $-100 \mathrm{mV}$. Commands to potentials more depolarized than $-50 \mathrm{mV}$ elicited a rapid TTXsensitive inward membrane current, which presumably reflects activation of the $\mathrm{Na}^{+}$conductance underlying the action potential. Depolarizing commands also evoked a slow outward current response that was suppressed by addition of $25 \mathrm{~mm}$ TEA to the bathing medium. This current primarily reflects activation of the 'TEA-sensitive delayed rectifier $\mathrm{K}^{+}$conductance $\left(I_{\mathrm{K}}\right)$ (Hodgkin and Huxley, 1952; Hille, 1967). Concentrations of TEA lower than $25 \mathrm{mM}$ were only partially effective in blocking $I_{\mathrm{K}}$. In the presence of TTX and TEA, a transient outward current remained during depolarizing voltage steps from holding potentials equal to or more negative than -60 $\mathrm{mV}$. These characteristics are superficially similar to those reported for $I_{A}$ in molluscan neurons (Connor and Stevens, 1971a; Neher, 1971), and, therefore, the corresponding transient outward current in these mammalian neurons will be identified as $I_{A}$ in this paper.

$I_{A}$ was recorded in 61 of 63 hippocampal neurons and in 30 of 35 spinal cord cells. The amplitude of the current transient increased rapidly upon stepping to more positive command potentials from a fixed holding potential (Fig. 1A1). At holding potentials equal to or more positive than $-50 \mathrm{mV}, I_{A}$ was completely inactivated, but brief hyperpolarizing conditioning prepulses could remove this inactivation. The amplitude of $I_{A}$ was strongly dependent upon the potential of the conditioning pulse, increasing toward a maximum as the conditioning potential reached $-100 \mathrm{mV}$ (Fig. 1A2). Complete reactivation at a given potential required conditioning pulses more than 200 msec in duration, while one-half recovery occurred with hyperpolarizing steps about $20 \mathrm{msec}$ in duration (Fig. $1 D$ ). The time to peak of the response varied from 3 to 10 msec (Fig. $1 A$ ) and the current decayed exponentially with a time constant ranging from 10 to $40 \mathrm{msec}$ (Fig. $1 C)$. This decay rate was independent of command potential over the range of potentials examined. In most cells the decay could be fitted by a single exponential (Fig. 1C), although in some neurons a second component with a much slower time constant ( $>100 \mathrm{msec}$ ) was detected.

Typical curves for inactivation, with normalized peak current amplitude plotted against holding potential, and for activation, with conductance calculated by dividing the current amplitude extrapolated to time zero by the driving force, are displayed in Figure $1 B . I_{A}$ is one-half inactivated at about $-75 \mathrm{mV}$.

The equilibrium potential of $I_{A}$ was determined by recording the tail currents occurring following a second voltage step at the peak of the current transient. The reversal potential of the tail current was -70 to $-75 \mathrm{mV}$ in five cells, and this reversal potential changed in a depolarizing direction when $\left[\mathrm{K}^{+}\right]_{0}$ was raised by ejecting medium containing $25 \mathrm{mM} \mathrm{KCl}$ in the vicinity of the cell (Fig. $1 E$ ).

As in other neuronal membranes (Thompson, 1977; Gustafsson et al., 1982), $I_{A}$ was depressed by externally applied 4-aminopyridine (4-AP) in a dose-dependent, reversible manner. Both the rate of depression by the drug and the rate of recovery from its effects were relatively slow, with full recovery requiring up to $10 \mathrm{~min}$ (Fig. 2A). Half-maximal depression of $I_{A}$ occurred with $2 \mathrm{mM} 4$-AP, whereas 80 to $90 \%$ blockade required $10 \mathrm{mM}$ (Fig. 2B). The blocking effects of 4-AP did not appear to be dependent on prior activation, since maximal blockade of $I_{A}$ could be observed during the first voltage step following application of the drug. As demonstrated in Figure 2C, 4-AP caused a uniform depression of $I_{A}$ throughout all command potentials.

In some experiments, the cations $\mathrm{Cd}^{2+}(0.2 \mathrm{~mm})$ or $\mathrm{Co}^{2+}(5 \mathrm{mM})$ were added to the recording medium to block $\mathrm{Ca}^{2+}$ entry. Under these conditions, transient outward currents could still be recorded, and these were sensitive to 4-AP, indicating that $I_{A}$ is not primarily dependent upon $\mathrm{Ca}^{2+}$ influx (see Adams et al., 1982a, and MacDermott and Weight, 1982) and that 4-AP does not influence $I_{A}$ through an alteration of $\mathrm{Ca}^{2+}$ fluxes (see Rogawski and Barker, 1983). Nevertheless, the ionic $\mathrm{Ca}^{2+}$ channel antagonists did have distinct effects on $I_{A}$ in a number of cells either to partially suppress or to enhance the current. A detailed analysis of the effects of $\mathrm{Ca}^{2+}$ 
A1

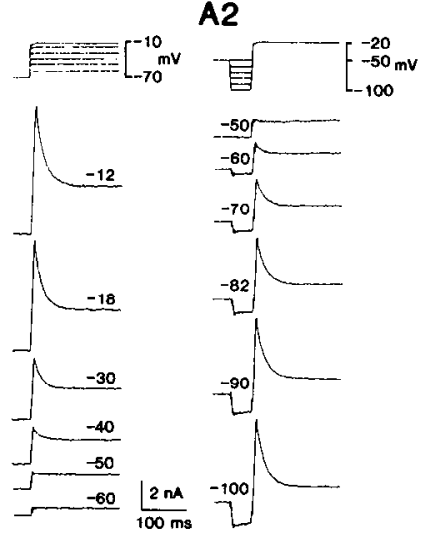

B

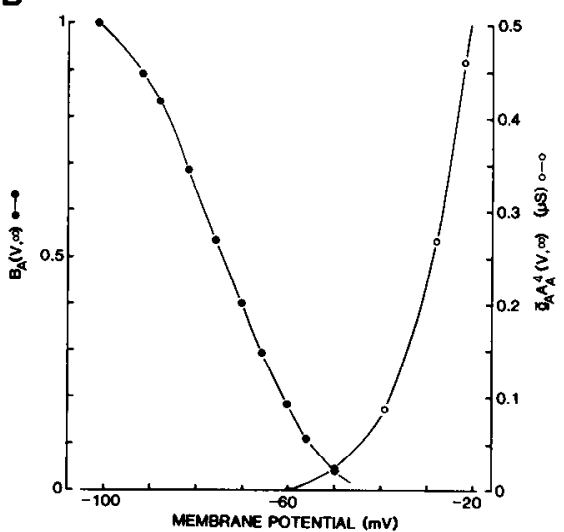

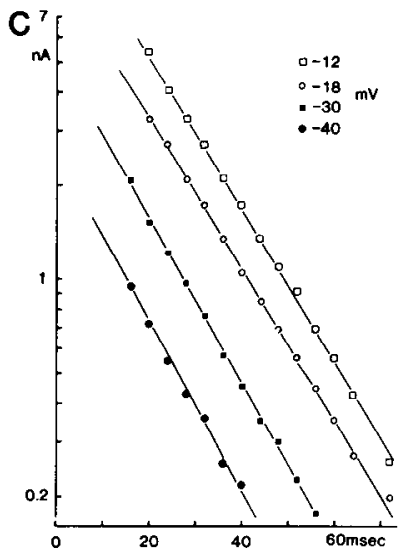
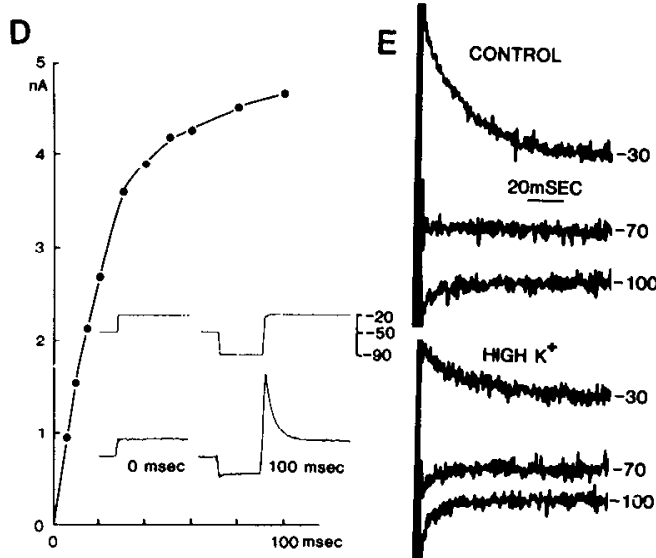

Figure 1. Voltage clamp analysis of $I_{A}$ in cultured spinal and hippocampal neurons bathed in a medium containing TTX $(1 \mu \mathrm{M})$ and TEA $(25 \mathrm{mM}) . A 1$, Pen recorder traces show rapid activation of a transient outward current as the membrane potential is stepped from $-70 \mathrm{mV}$ to depolarized potentials in a cultured mouse spinal neuron. A2, Hyperpolarizing conditioning pulses 50 msec in duration remove inactivation obtained by holding at $-20 \mathrm{mV}$. $B$, Data similar to those illustrated in $A$ have been used to construct activation and inactivation curves (see "Materials and Methods"). $C$, Semilogarithmic plots demonstrating the exponential decay of $I_{A}$. The time constant $(\sim 24 \mathrm{msec})$ is independent of voltage. $D$, Relationship between the duration of the conditioning pulse and the degree of activation of $I_{A}$. Although $I_{A}$ recovers by $50 \%$ in about $20 \mathrm{msec}$, it is not fully recovered until at least $200 \mathrm{msec}$ (not shown). $E$, The reversal potential of $I_{A}$ was measured in a hippocampal neuron using a double-step protocol. The cell was held at $-70 \mathrm{mV}$, stepped first to $-20 \mathrm{mV}$ for 20 msec and then stepped back to various potentials. The records shown are the tail currents recorded upon returning to the indicated potentials. The control records (top), obtained in bathing medium containing $5 \mathrm{~mm}\left[\mathrm{~K}^{+}\right]_{0}$, show a reversal potential for the tail current of $-75 \mathrm{mV}$. The reversal potential moves in a depolarizing direction when $\left[\mathrm{K}^{+}\right]_{0}$ is elevated by applying $25 \mathrm{~mm}$ $\mathrm{KCl}$ in the vicinity of the cell (bottom).

entry blockers on $I_{A}$ was not carried out in the present study.

The effects of 4-AP and TEA were compared in experiments carried out in bathing medium to which TEA had not been added. 4-AP had a relatively selective depressant effect on the initial outward current transient, whereas TEA predominately blocked the late outward current, although minimal suppression of $I_{A}$ was occasionally observed (Fig. $2 C$, inset). Often 4-AP caused an increase in the total delayed current, an effect which might be attributed to its enhancement of the $\mathrm{Ca}^{2+}$. dependent $\mathrm{K}^{+}$conductance (Hermann and Gorman, 1981; Rogawski and Barker, 1983).

The functional significance of the transient outward current was evaluated by studying the effects of 4-AP on the membrane potential behavior of current-clamped spinal cord and hippocampal neurons. The current-voltage properties of the cells were examined by injecting a square current pulse through a single recording electrode using a bridge circuit. In the presence of TTX, TEA, and $\mathrm{Co}^{2+}$, transient rectification of the membrane was noted when the voltage excursion produced by such a pulse was 

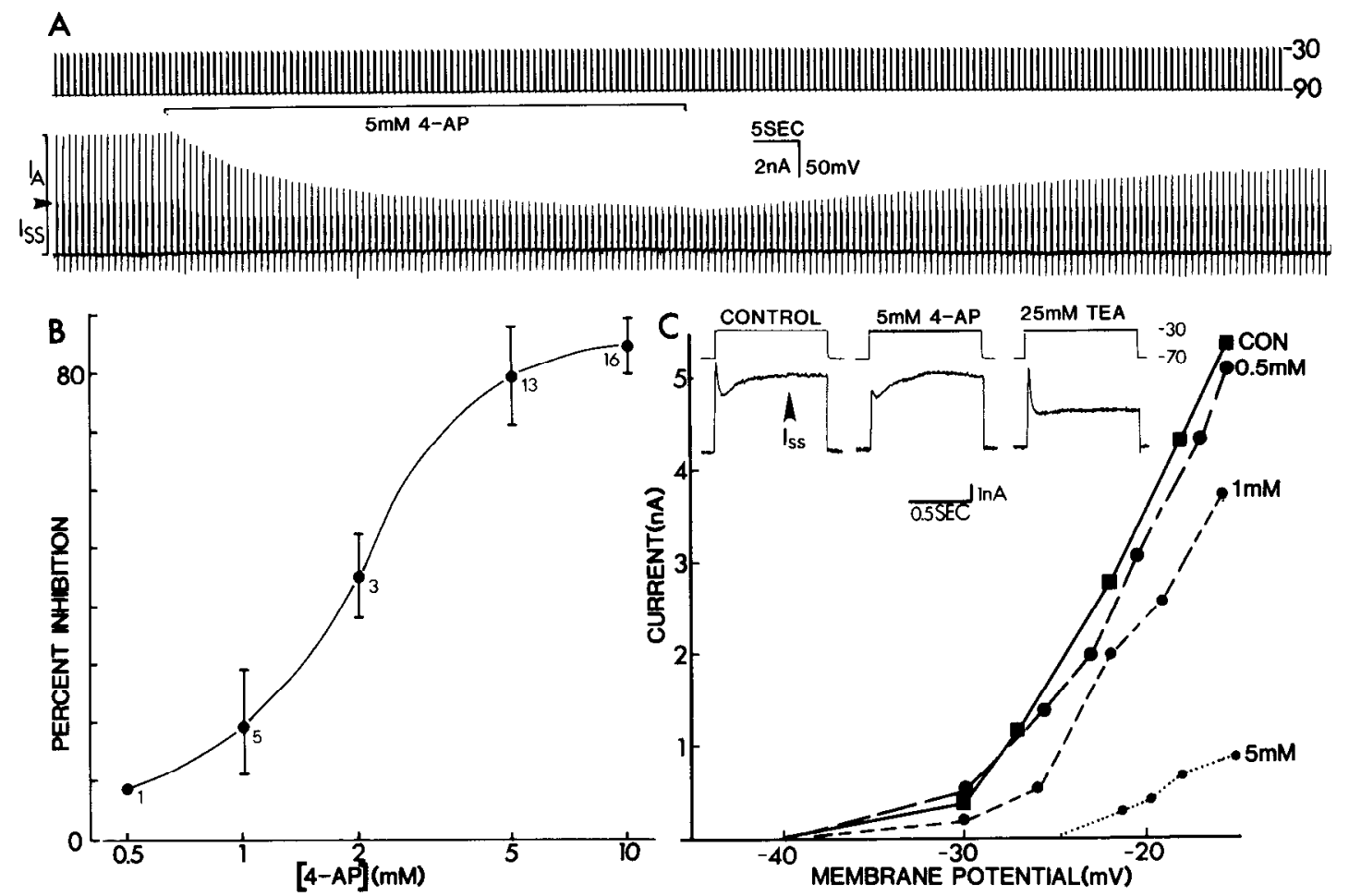

Figure 2. 4-AP reduces $I_{A}$ in hippocampal and spinal cord cells. The recording medium used in these experiments contained TTX $(1 \mu \mathrm{M})$, TEA $(25 \mathrm{mM})$, and $\mathrm{Cd}^{2+}(0.2 \mathrm{mM})$. A, Continuous pen recorder traces show repeated 100-msec voltage steps to -30 $\mathrm{mV}$ from a holding potential of $-90 \mathrm{mV}$ (above) and the resulting current responses (below) before, during, and after the application of 4-AP ( $5 \mathrm{mM}$ ). The drug markedly reduces the peak amplitude of $I_{A}$ (thin, upward deflections, delineated by the arrowhead at the left) and slightly decreases the steady-state current response $I_{s s}$ (thick upward deflections) in a time-dependent, slowly reversible manner. $B$, Dose response curve for inhibition of $I_{A}$ as a function of 4-AP concentration. The concentration dependencies of the effects of 4-AP on $I_{A}$ in hippocampal and spinal cord cells were similar; therefore, the data derived from experiments in both cell types have been combined. Numerals beside each data point indicate number of cells tested. $C$, Plots of $I_{A}$ amplitude in a hippocampal neuron as a function of membrane potential under control conditions and in the presence of increasing concentrations of 4-AP. The inset shows the selective depressant effects of 4 -AP and TEA on $I_{A}$ and the delayed rectifier current, $I_{\mathrm{K}}$, respectively.

equal to or more positive than $-45 \mathrm{mV}$ (Fig. $3 A$, top panel). 4-AP (5 mM) attenuated this rectification, thus allowing the initial phase of the electronic potential to reach more depolarized levels (Fig. $3 A$, bottom panel). In media free of TTX and TEA (but containing $5 \mathrm{mM} \mathrm{Co}^{2+}$ ), 4-AP decreased the threshold potential required for triggering single and repetitive action potentials (Fig. 3, $B$ and $C$ ). In the presence of the drug, action potentials were triggered at more hyperpolarized levels using less depolarizing current. For example, $5 \mathrm{~mm}$ 4-AP lowered the threshold for triggering an action potential from $-39.4 \pm 1.3 \mathrm{mV}$ to $-45.1 \pm 1.6 \mathrm{mV}$ (mean \pm SEM) in nine hippocampal cells. In some cells, repetitive activity could not be triggered at any level of injected current until 4-AP was applied. In many cells, 4-AP produced no change in the action potential duration, although occasionally some spike broadening did occur. On the other hand, TEA invariably produced marked spike broadening but usually had minimal, if any, effects on action potential threshold (Fig. 3C, inset).

\section{Discussion}

Cultured vertebrate central neurons demonstrate a transient voltage-dependent outward current carried by potassium. Although present in most neurons cultured from embryonic spinal cord and hippocampus, the current is not found in all types of cultured mammalian neuron, being noticeably absent from sensory cells derived from dorsal root ganglia (unpublished observations). In recent experiments, we have detected the current in cultured hypothalamic neurons as well. The current is distinct from other potassium currents of vertebrate neuron somata, such as the delayed rectifier (Adams et al., 1982b), the $\mathrm{Ca}^{2+}$-activated $\mathrm{K}^{+}$current (Meech, 1978; Brown and Griffith, 1983), and the M-current (Brown and Adams, 1980; Adams et al., 1982b).

The transient outward current resembles $I_{A}$ of molluscan neurons in its underlying ionic basis, voltage dependency, kinetics, and pharmacological sensitivity (Connor and Stevens, 1971a; Neher, 1971; Thompson, 1977). As in molluscan neurons, $\mathrm{K}^{+}$is likely to be the predominant charge carrier of the current. Since $I_{A}$ is always outward, its equilibrium potential must be more negative than potentials at which it is activated, i.e., about $-50 \mathrm{mV}$. Analysis of the tail current in a two-step protocol indicates that the reversal potential for $I_{A}$ is -70 to $-75 \mathrm{mV}$, near the expected equilibrium potential for $\mathrm{K}^{+}$. Moreover, raising $\left[\mathrm{K}^{+}\right]_{0}$ shifts the reversal potential in the depolarized direction, providing qualitative confirmation that $\mathrm{K}^{+}$is the charge carrier for the current.

The voltage dependency of $I_{A}$ in cultured mammalian 


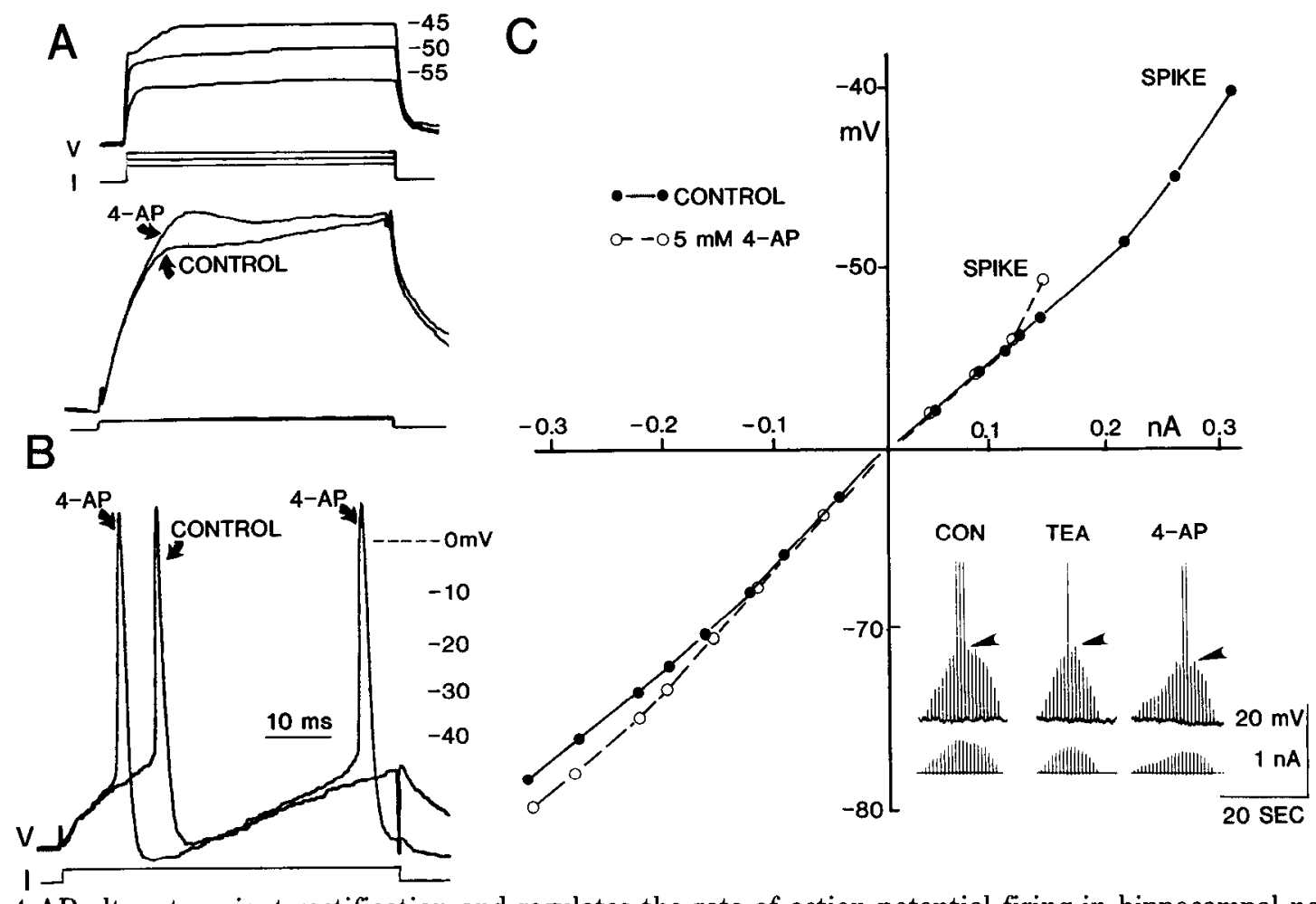

Figure 3. 4-AP alters transient rectification and regulates the rate of action potential firing in hippocampal neurons under current clamp. A, Top panel: Family of voltage and current records for a cell depolarized to various potentials by 500 -msec current pulses in medium containing TTX. Transient rectification lasting less than $100 \mathrm{msec}$ is evident at the beginning of the voltage response only when the potential becomes more positive than $-50 \mathrm{mV}$. Bottom panel: The transient rectification activated during a 50 -msec depolarizing pulse is blocked by 4 -AP $(5 \mathrm{mM})$ with little change in the voltage response developed at the end of the current pulse. $B$ and $C$, Recordings made from hippocampal cells in medium free of TTX or TEA but containing $\mathrm{Co}^{2+}(5 \mathrm{mM}) . B$ shows the response of a cell held at $-60 \mathrm{mV}$ to 50 -msec depolarizing current pulses $(0.3 \mathrm{nA}) .4-\mathrm{AP}(5 \mathrm{mM})$ shifts the potential at which an action potential is triggered from $-43 \mathrm{mV}$ to $-48 \mathrm{mV}$ and induces repetitive discharge. The action potentials triggered in 4-AP do not exhibit detectable changes in either peak amplitude or duration. $C$, Current-voltage plot of voltage responses to 50-msec hyperpolarizing and depolarizing current pulses, before and during application of 4-AP (5 mM). 4AP lowers the spike threshold by about $10 \mathrm{mV}$ without appreciably changing the steady-state current-voltage relationship. Inset, Pen recorder traces showing a series of voltage responses to a family of 50-msec depolarizing current stimuli. 4 -AP (5 mM) but not TEA (25 mM) lowers spike threshold (arrowheads).

neurons is remarkably similar to that in Anisodoris (Connor and Stevens, 1971a) or Tritonia (Thompson, 1977) where $I_{A}$ is activated upon stepping from hyperpolarized levels to -50 to $-40 \mathrm{mV}$ but is inactivated at potentials more positive than $-50 \mathrm{mV}$. In these invertebrate neurons, as in cultured mammalian neurons, $I_{A}$ is one-half inactivated at approximately -70 to $-75 \mathrm{mV}$. In contrast to our results and those obtained in invertebrates, in sympathetic neurons the threshold for activation and the potential at which inactivation is removed may be at slightly more hyperpolarized levels (Adams et al., 1982b; Galvan, 1982).

The kinetic parameters of $I_{A}$ in molluscan neurons are an order of magnitude slower than for cultured mammalian neurons, in part attributable to the lower temperature of the invertebrate experiments. Nevertheless, the general kinetic properties of $I_{A}$ are conserved. Thus, in all systems, the rise of $I_{A}$ is more rapid than its decay; however, due to limilations of our voltage clamp it was not possible to obtain an accurate determination of the activation kinetics and a sigmoidal activation characteristic could not be confirmed (see Conner and Stevens, 1971a). Moreover, across phylogeny $I_{A}$ decays exponentially with a time constant that is for the most part insensitive to voltage. It should be recognized that the present experiments were carried out at room temperature to allow better resolution of the fast transient current and that the absolute kinetic parameters will differ at body temperature.

4-AP produced a selective blockade of $I_{A}$ with an $\mathrm{IC}_{50}$ of about $2 \mathrm{mM} . I_{A}$ in Tritonia neurons shows a similar pharmacological sensitivity to 4-AP with an essentially superimposable dose response curve (Thompson, 1977). In contrast, in $\mathrm{CA}_{3}$ hippocampal neurons some blockade of $I_{A}$ was noted at $100 \mu \mathrm{M}$ (Gustafsson et al., 1982), and in rat sympathetic neurons the current was markedly reduced at $1 \mathrm{mM}$ (Galvan, 1982). On the other hand, little or no effect was reported for bullfrog sympathetic neurons at $1 \mathrm{mM}$ (Adams et al., 1982b). Thus, among vertebrate neurons there may be some variability in sensitivity to 4-AP, although methodological differences are likely to account for much of this discrepancy. It should be noted that the pressure ejection technique we used does not allow the exact concentration at the cell membrane to be determined precisely, although it does define an upper limit for this value.

Despite the similarities in the transient outward current described here and that of molluscs, the complex geometry of cultured mammalian neurons contrasts sharply with that of invertebrate somata, and the ques- 
tion of the adequacy of voltage control must be raised. Although it has not been possible to measure directly the quality of the space clamp obtained with our two-electrode system, an analysis of voltage responses to constant current pulses indicates that the cells are electrically compact (see Segal, 1983). Nevertheless, quantitative parameters must be considered approximate.

Although we recorded a 4-AP-sensitive transient outward current from most spinal and hippocampal neurons, there were some differences between neurons cultured from the two regions. Namely, the magnitude and decay time constant of $I_{A}$ among spinal neurons spanned a wide range of values, whereas this variability was not encountered in hippocampal neurons.

In conclusion, the unique voltage sensitivity and kinetics of $I_{A}$ and the ability to effect its pharmacological separation from the total potassium current suggest the existence of a distinct population of $\mathrm{K}^{+}$selective channels which carry $I_{A}$. Moreover, the high degree of pharmacological specificity displayed by 4-AP and TEA emphasizes that the drugs cannot be considered interchangeable $\mathrm{K}^{+}$channel blockers. At concentrations less than $10 \mathrm{mM}, 4-\mathrm{AP}$ rather selectively blocks $I_{A}$ but not $I_{\mathrm{K}}$; as a consequence, spike threshold is reduced, but spike broadening is not prominent. Conversely, $25 \mathrm{mM}$ TEA markedly suppresses $I_{\mathrm{K}}$ with little influence on $I_{A}$, so that threshold remains constant but spikes are markedly broadened.

The ability of 4-AP to selectively block $I_{A}$ allows the drug to be used to investigate the functional role of the current. Under unclamped conditions but with action potentials blocked by TTX, the rise of the electrotonic potential recorded in response to injected current is retarded for about $50 \mathrm{msec}$ by an active membrane process. This transient rectification is present only when the membrane potential reaches voltages where $I_{A}$ is activated. The observation that 4-AP attenuates this transient rectification indicates that it is a reflection of $I_{A}$ activation. Under normal conditions, $I_{A}$ could be turned on during repolarization from the afterhyperpolarization phase of an action potential or during rapid depolarizing synaptic events. In either of these circumstances, $I_{A}$ would tend to retard further action potential generation and thus dampen excitability. Interference with $I_{A}$ should lead to abnormal neuronal discharge, and this might, in part, contribute to the convulsant activity of 4-AP. Moreover, physiological modulation of $I_{A}$ could be an important factor in regulating neuronal firing in the mammalian central nervous system.

\section{References}

Adams, D. J., S. J. Smith, and S. H. Thompson (1980) Ionic currents in molluscan soma. Annu. Rev. Neurosci. 3: 141167.

Adams, P. R., A. Constanti, D. A. Brown, and R. B. Clark (1982a) Intracellular $\mathrm{Ca}^{2+}$ activates a fast voltage-sensitive $\mathrm{K}^{+}$current in vertebrate sympathetic neurones. Nature 296: $746-749$.

Adams, P. R., A. Constanti, and D. A. Brown (1982b) MCurrents and other potassium currents in bullfrog sympathetic neurons. J. Physiol. (Lond.) 330: 537-572.
Alkon, D. L., I. Lederhendler, and J. L. Shoukimas (1982) Primary changes of membrane currents during retention of associative learning. Science 215: 693-695.

Barker, J. L., and M. A. Rogawski (1982) Identification and pharmacological sensitivity of a transient outward current in the somata of cultured mammalian central neurons. Soc. Neurosci. Abstr. 8: 123.

Barker, J. L., R. N. McBurney, and J. F. MacDonald (1982) Fluctuation analysis of neutral amino acid responses in cultured mouse spinal neurons. J. Physiol. (Lond.) 322: 365387.

Brown, D. A., and P. R. Adams (1980) Muscarinic suppression of a novel voltage-sensitive $\mathrm{K}^{+}$current in a vertebrate neurone. Nature 283: 673-676.

Brown, D. A., and W. H. Griffith (1983) Calcium-activated outward current in voltage-clamped hippocampal neurons of the guinea-pig. J. Physiol. (Lond.) 337: 287-301.

Connor, J. A., and C. F. Stevens (1971a) Voltage clamp studies of a transient outward membrane current in gastropod neural somata. J. Physiol. (Lond.) 213: 21-30.

Connor, J. A., and C. F. Stevens (1971b) Prediction of repetitive firing behaviour from voltage clamp data on an isolated neurone somata. J. Physiol. (Lond.) 213: 31-53.

Galvan, M. (1982) A transient outward current in rat sympathetic neurones. Neurosci. Lett. 31: 295-300.

Gustafsson, B., M. Galvan, P. Grafe, and H. Wigstrom (1982) A transient outward current in a mammalian central neurone blocked by 4-aminopyridine. Nature 299: 252-254.

Hermann, A., and A. L. F. Gorman (1981) Effects of 4-aminopyridine on potassium currents in a molluscan neuron. J. Gen. Physiol. 78: 63-86.

Hille, B. (1967) The selective inhibition of delayed potassium currents in nerve by tetraethylammonium ion. J. Gen. Physiol. 50: 1287-1307.

Hodgkin, A. L., and A. F. Huxley (1952) A quantitative description of membrane current and its application to conduction and excitation in nerves. J. Physiol. (Lond.) 117: 500-544.

MacDermott, A. B., and F. F. Weight (1982) Action potential repolarization may involve a transient, $\mathrm{Ca}^{2+}$-sensitive outward current in a vertebrate neurone. Nature 300: 185-188.

Meech, R. W. (1978) Calcium-dependent potassium activation in nervous tissue. Annu. Rev. Biophys. Bioeng. 7: 1-18.

Neher, E. (1971) Two fast transient current components during voltage clamp on snail neurons. J. Gen. Physiol. 58: 36-53.

Ransom, B. R., E. Neale, M. Henkart, P. N. Bullock, and P. G. Nelson (1977) Mouse spinal cord in cell culture. I. Morphology and intrinsic neuronal electrophysiologic properties. J. Neurophysiol. 40: 1132-1150.

Rogawski, M. A., and J. L. Barker (1983) Effects of 4-aminopyridine on calcium action potentials and calcium current under voltage clamp in spinal neurons. Brain Res. 280: 180-185.

Segal, M. (1983) Rat hippocampal neurons in culture: Responses to electrical and chemical stimuli. J. Neurophysiol. 50: $1249-1264$.

Segal, M., and J. L. Barker (1982) Voltage clamp analysis of cultured rat hippocampal neurons. Soc. Neurosci. Abstr. 8: 800.

Shimahara, T. (1981) Modulation of synaptic output by the transient outward potassium current in Aplysia. Neurosci. Lett. 24: 139-142.

Smith, T. G., J. L. Barker, B. M. Smith, and T. R. Colburn (1980) Voltage clamping with microelectrodes. J. Neurosci. Methods 3: 105-128.

Thompson, S. H. (1977) Three pharmacologically distinct potassium channels in molluscan neurones. J. Physiol. (Lond.) 265: 465-488. 